\title{
Extended-pulsed fidaxomicin versus vancomycin for Clostridium difficile infection: EXTEND study subgroup analyses
}

\author{
Oliver A. Cornely ${ }^{1,2} \cdot$ Maria J. G. T. Vehreschild ${ }^{1} \cdot$ Nicholas Adomakoh $^{3} \cdot$ Areti Georgopali $^{4} \cdot$ Andreas Karas $^{5}$. \\ Gbenga Kazeem $^{3,6} \cdot$ Benoit Guery $^{7}$
}

Received: 28 November 2018 / Accepted: 28 February 2019 / Published online: 25 March 2019

(C) The Author(s) 2019

\begin{abstract}
Poor outcomes following Clostridium difficile infection (CDI) have been associated with advanced age, presence of cancer and C. difficile PCR-ribotype 027 . The impact of baseline risk factors on clinical outcomes was evaluated using data from the EXTEND study, in which rate of sustained clinical cure (SCC) in the overall population was significantly higher with an extended-pulsed fidaxomicin (EPFX) regimen than with vancomycin. Patients aged $\geq 60$ years received EPFX (fidaxomicin $200 \mathrm{mg}$ twice daily, days 1-5; once daily on alternate days, days 7-25) or vancomycin (125 mg four times daily, days 1-10). We analysed outcomes by advanced age, cancer diagnosis, CDI severity, prior CDI occurrence and infection with PCR-ribotype 027. The primary endpoint was SCC 30 days after end of treatment (EOT; clinical response at test-of-cure with no subsequent recurrence). SCC rates 30 days after EOT did not differ significantly between EPFX (124/177, 70.1\%) and vancomycin (106/ $179,59.2 \%)$ regardless of age, cancer diagnosis, CDI severity and prior CDI. In patients with PCR-ribotype 027, SCC rate 30 days after EOT was significantly higher with EPFX (20/25, 80\%) than with vancomycin $(9 / 22,40.9 \%)$ (treatment difference, $39.1 \%$; $95 \%$ CI, 13.2-64.9; $P=0.006)$. Subgroup analyses from the EXTEND study suggest that EPFX is efficacious as a potential treatment for CDI regardless of age, cancer diagnosis, infection with PCR-ribotype 027, CDI severity or prior CDI. ClinicalTrials.gov identifier: NCT02254967.
\end{abstract}

Keywords Clostridium difficile infection $\cdot$ Randomised controlled trial $\cdot$ Antibacterial agents $\cdot$ Cohort analyses $\cdot$ Recurrence

Electronic supplementary material The online version of this article (https://doi.org/10.1007/s10096-019-03525-y) contains supplementary material, which is available to authorized users.

Oliver A. Cornely

oliver.cornely@uk-koeln.de

Maria J. G. T. Vehreschild

maria.vehreschild@uk-koeln.de

1 Department I of Internal Medicine, University Hospital of Cologne and German Centre for Infection Research, Partner Site Bonn-Cologne, 50937 Cologne, Germany

2 Cologne Excellence Cluster on Cellular Stress Responses in Aging-Associated Diseases (CECAD), Clinical Trials Centre Cologne (ZKS Köln), University of Cologne, Cologne, Germany

3 Astellas Pharma, Inc., Chertsey, UK

4 Astellas Pharma Europe Ltd., Chertsey, UK

5 Astellas Pharma Ltd.,, Chertsey, UK

6 BENKAZ Consulting Ltd., Cambridge, UK

7 University Hospital and University of Lausanne, Lausanne, Switzerland

\section{Introduction}

Outcomes following Clostridium difficile infection (CDI) are especially poor in elderly patients, patients with severe disease and those with cancer: these patient groups experience higher rates of CDI complications and/or recurrence [1-4]. Additionally, specific C. difficile strains linked to infection outbreaks, including PCR-ribotype 027, are associated with severe CDI sequelae compared with other strain types [5-10].

CDI recurrence occurs in approximately $24-27 \%$ of cases treated with vancomycin or metronidazole [11] and may be a consequence of disruption and delayed recovery of the natural gut microbiota following antibiotic treatment [12]. Fidaxomicin is associated with both greater conservation of the gut microbiota following CDI treatment [13] and significantly lower recurrence rates than vancomycin $[14,15]$. Furthermore, an extended-pulsed fidaxomicin (EPFX) regimen, which extends administration of the 20 tablets of a regular regimen from 10 to 25 days, may enable fidaxomicin to persist in the gut at 
inhibitory concentrations, thereby suppressing $C$. difficile and facilitating microbiota recovery [16].

In the overall EXTEND study population of patients aged $\geq 60$ years, EPFX provided significantly superior rates of sustained clinical cure (SCC) compared with standard vancomycin [17]. The present study evaluated clinical outcomes in the EXTEND study population according to presence of cancer, advanced age, CDI severity, prior CDI episodes and presence of $C$. difficile PCR-ribotype 027.

\section{Materials and methods}

\section{Study design and patients}

EXTEND was an open-label, randomised, active-comparator controlled, multicentre, phase $3 \mathrm{~b} / 4$ study conducted in
Europe. The study design and primary results have been reported elsewhere [17]. Enrolled patients were aged $\geq 60$ years and hospitalised with clinically confirmed CDI, as defined previously [17].

\section{Treatment and assessments}

Patients in the EXTEND study received EPFX (200 mg oral fidaxomicin twice daily on days $1-5$, then oncedaily administration on alternate days on days 7-25) or vancomycin (125 mg orally, four times daily on days 1-10) [17]. Clinical response, CDI recurrence and safety were assessed as described previously [17]. SCC was defined as clinical response at test-of-cure (TOC) with no subsequent CDI recurrence. PCR ribotyping of all $C$. difficile isolates from stool samples was performed at a central laboratory (Leeds Institute of Biomedical \&

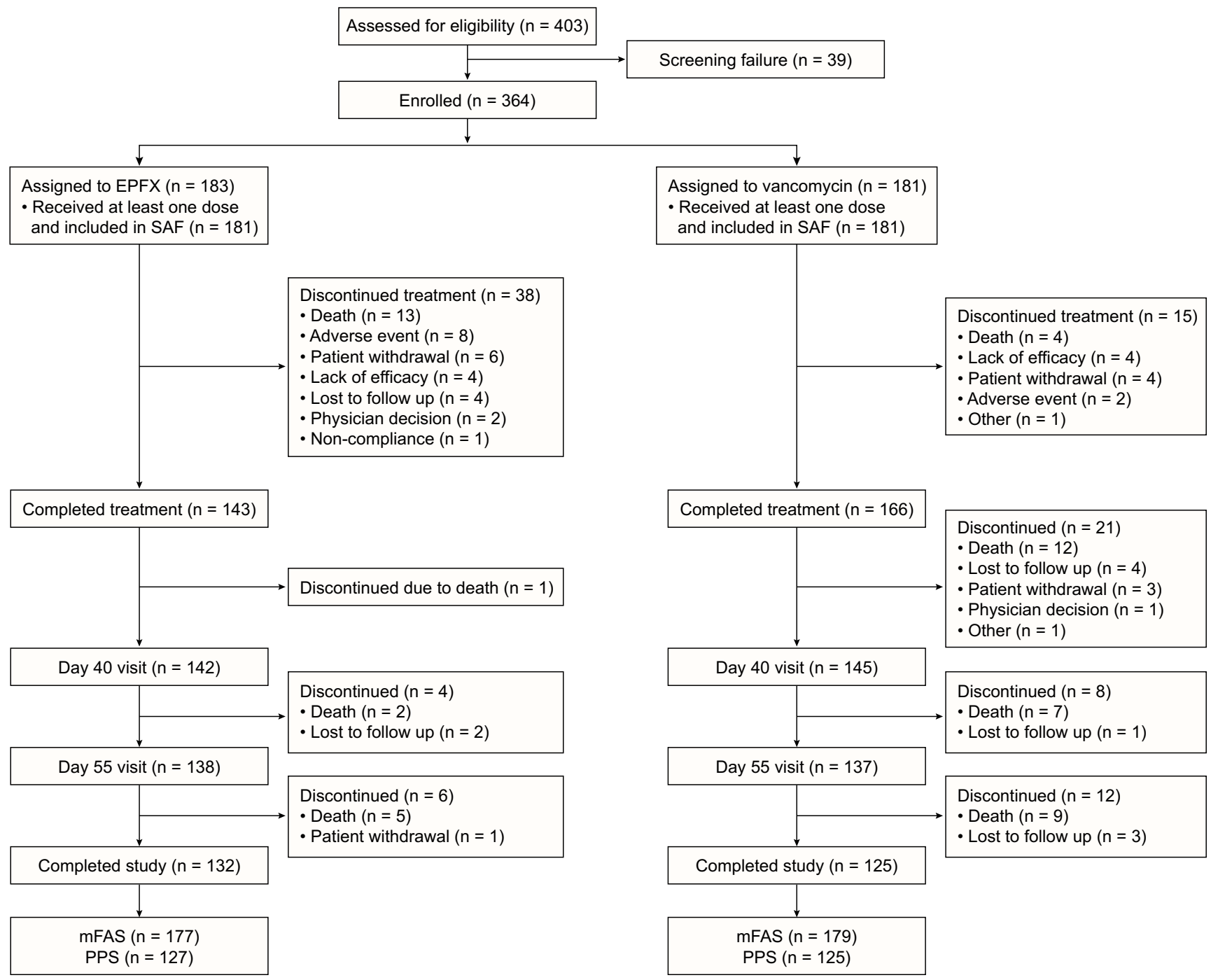

Fig. 1 Patient flow through the study 
Clinical Sciences, University of Leeds, Leeds, UK), using capillary gel electrophoresis $[18,19]$.

\section{Statistical analysis}

Efficacy evaluations and analysis sets have been described previously [17]. In the present study, prespecified subgroup analyses assessed efficacy endpoints stratified by the following baseline characteristics: patient age (60-74 years versus $\geq 75$ years); cancer diagnosis (presence versus absence); CDI severity (according to European Society of Clinical Microbiology and Infectious Diseases [ESCMID] criteria $[5,17]$ ) and number of prior CDI episodes within 3 months before study participation. A post hoc subgroup efficacy analysis was performed in relation to presence of $C$. difficile PCR-ribotype 027 (versus other ribotypes). For each subgroup assessment, the Cochran-Mantel-Haenszel $(\mathrm{CMH})$ test was performed, adjusted for other baseline stratification factors. Common odds ratios, corresponding $95 \%$ CIs and descriptive $P$ values (significance at $\leq 0.05$ ) were calculated using the $\mathrm{CMH}$ test, or the chi-square test for PCR-ribotype assessments.

\section{Results and discussion}

Of the 364 patients randomised (Fig. 1), 356 were included in the modified full analysis set (mFAS, comprising all
Table 1 Demographics and baseline characteristics of participants, mFAS

\begin{tabular}{|c|c|c|c|}
\hline Characteristic & $\operatorname{EPFX}(n=177)$ & Vancomycin $(n=179)$ & Total $(n=356)$ \\
\hline \multicolumn{4}{|l|}{ Gender, $n(\%)$} \\
\hline Female & $107(60.5)$ & $100(55.9)$ & $207(58.1)$ \\
\hline \multicolumn{4}{|l|}{ Race, $n(\%)^{\mathrm{a}}$} \\
\hline White & $149(84.2)$ & $153(85.5)$ & $302(84.8)$ \\
\hline Missing & $28(15.8)$ & $26(14.5)$ & $54(15.2)$ \\
\hline Median (range) age, years & $75.0(60-94)$ & $75.0(60-95)$ & $75.0(60-95)$ \\
\hline \multicolumn{4}{|l|}{ UBMs per day, $n^{\mathrm{b}}$} \\
\hline Mean (SD) & $6.8(4.7)$ & $6.4(3.4)$ & $6.6(4.1)$ \\
\hline Median & 5.0 & 5.0 & 5.0 \\
\hline Severe CDI at baseline, $n(\%)^{\mathrm{c}}$ & $63(35.6)$ & $67(37.4)$ & $130(36.5)$ \\
\hline Severe CDI by ESCMID score, $n(\%)$ & $78(44.1)$ & $84(46.9)$ & $162(45.5)$ \\
\hline \multicolumn{4}{|c|}{ No. previous CDI occurrences in the past 3 months, $n(\%)$} \\
\hline 0 & $141(79.7)$ & $140(78.2)$ & $281(78.9)$ \\
\hline 1 & $26(14.7)$ & $29(16.2)$ & $55(15.4)$ \\
\hline 2 & $10(5.6)$ & $10(5.6)$ & $20(5.6)$ \\
\hline Cancer present, $n(\%)^{\mathrm{c}}$ & $38(21.5)$ & $37(20.7)$ & $75(21.1)$ \\
\hline \multicolumn{4}{|c|}{ Use of antibiotics for condition other than CDI, $n(\%)$} \\
\hline Yes & $128(72.3)$ & $129(72.1)$ & $257(72.2)$ \\
\hline Residential setting, $n(\%)$ & $n=175$ & $n=179$ & $n=354$ \\
\hline Own residence & $102(58.3)$ & $103(57.5)$ & $205(57.9)$ \\
\hline Family residence & $66(37.7)$ & $59(33.0)$ & $125(35.3)$ \\
\hline Nursing home & $4(2.3)$ & $6(3.4)$ & $10(2.8)$ \\
\hline Long-term care facility & $1(0.6)$ & $4(2.2)$ & $5(1.4)$ \\
\hline Other & $2(1.1)$ & $7(3.9)$ & $9(2.5)$ \\
\hline Missing & 2 & 0 & 2 \\
\hline \multicolumn{4}{|l|}{ C. difficile PCR-ribotype } \\
\hline 027 & $25(14.1)$ & $22(12.3)$ & $47(13.2)$ \\
\hline Other & $152(85.9)$ & $157(87.7)$ & $309(86.8)$ \\
\hline
\end{tabular}

$E P F X$, extended-pulsed fidaxomicin; $m F A S$, modified full analysis set (all patients with confirmed CDI who were randomised and received at least one dose of study medication); $S D$, standard deviation; UBMS, unformed bowel movements

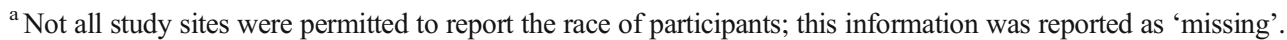

${ }^{\mathrm{b}}$ In the last $24 \mathrm{~h}$ prior to randomisation. ${ }^{\mathrm{c}}$ As provided in the Interactive Web Response System at randomisation, and defined as leukocyte count $>15 \times 109 / \mathrm{L}$ or rise in serum creatinine $>50 \%$ above the patient's normal level or albumin $<30 \mathrm{~g} / \mathrm{L}$ 
randomised patients who met the inclusion criteria and received $\geq 1$ dose of study medication). The treatment groups had similar baseline characteristics (Table 1) [17].

\section{Clinical outcomes}

Rates of SCC at 30 days after end of treatment (EOT) (the primary endpoint) did not differ significantly between EPFX and standard vancomycin treatment in most of the subgroups analysed; however, in patients with $C$. difficile PCR-ribotype 027, rates were significantly higher with EPFX than vancomycin (Table 2).

SCC rates at days 40 and 90 were significantly higher with EPFX than with standard vancomycin in patients aged $\geq 75$ years (Online Resource; ESM Fig. 1a). SCC rates at other time points and clinical response rates at day 12 and 2 days after EOT did not differ between treatments for either age category (Online Resource; ESM Fig. 1a and 1b). Our findings are supported by a previous study that used regression modelling to test the effects of age on treatment outcomes in phase 3 trials of standard-regimen fidaxomicin: a significantly higher probability of SCC was shown with fidaxomicin than standard vancomycin (odds ratio, 1.86; 95\% CI, 1.40-2.47; $P<0.001)$. However, irrespective of treatment choice, the probability of SCC decreased by $13 \%$ and the risk of recurrence increased by $17 \%$ for each decade increase in age [20].
At day 40, rates of SCC were significantly higher with EPFX than with vancomycin in both the severe and non-severe CDI subgroups. At days 55 and 90, SCC rates were significantly higher with EPFX than vancomycin only in the non-severe CDI subgroup (Online Resource; ESM Fig. 2a). There were no significant between-treatment differences in efficacy at other time points in patients with severe and non-severe CDI (Online Resource; ESM Fig. 2b).

Patients with a previous history of CDI are at greater risk of developing a further CDI episode [21]. Seventyfive $(21 \%)$ patients in our analysis had experienced a recent CDI episode prior to study enrolment; the majority (55 [15\%]) had a single prior episode. Rates of SCC at days 40, 55 and 90 were significantly higher with EPFX than with vancomycin in patients who had no prior occurrence of CDI. Additionally, rates of SCC at day 40 were significantly higher with EPFX in patients with one or two prior CDI episodes (Online Resource; ESM Fig. 3a). Other efficacy endpoints did not differ between treatments regardless of number of prior CDI episodes (Online Resource; ESM Fig. 3a and 3b). In a previous subgroup analysis, the 28-day SCC rate among patients with one prior CDI episode was $80.3 \%$ with standard fidaxomicin and $64.5 \%$ with standard vancomycin, higher than the rates observed in our analyses; this difference may be due to the younger age (median 63 years) of the patients in the previous analysis [22]. Our conclusions are also limited by the low number of patients in our study with

Table 2 Rates of sustained clinical cure of Clostridium difficile infection (CDI) at 30 days after end of treatment with extended-pulsed fidaxomicin (EPFX) and vancomycin in subgroups stratified by baseline characteristics, mFAS

\begin{tabular}{|c|c|c|c|}
\hline \multirow[t]{2}{*}{ Subgroup } & \multicolumn{2}{|c|}{ Sustained clinical cure of CDI 30 days after EOT $(\% ; 95 \% \mathrm{CI})$} & \multirow[t]{2}{*}{ Treatment difference $(95 \% \mathrm{CI}) ; P$ value } \\
\hline & $\operatorname{EPFX}(N=177)$ & Vancomycin $(N=179)$ & \\
\hline Age $\geq 75$ years & $66 / 97(68.0 ; 58.8,77.3)$ & $55 / 97(56.7 ; 46.8,66.6)$ & $11.3(-2.2,24.9) ; P=0.224$ \\
\hline Age $<75$ years & $58 / 80(72.5 ; 62.7,82.3)$ & $51 / 82(62.2 ; 51.7,72.7)$ & $10.3(-4.0,24.7) ; P=0.371$ \\
\hline Severe CDI & $39 / 63(61.9 ; 49.9,73.9)$ & $34 / 67(50.7 ; 38.8,62.7)$ & $11.2(-5.8,28.1) ; P=0.235$ \\
\hline Non-severe CDI & $85 / 114(74.6 ; 66.6,82.6)$ & $72 / 112(64.3 ; 55.4,73.2)$ & $10.3(-1.7,22.2) ; P=0.068$ \\
\hline No previous CDI episode & $99 / 141(70.2 ; 62.7,77.8)$ & $88 / 140(62.9 ; 54.9,70.9)$ & $7.4(-3.6,18.4) ; P=0.166$ \\
\hline One previous CDI episode & $17 / 26(65.4 ; 47.1,83.7)$ & $14 / 29(48.3 ; 30.1,66.5)$ & $17.1(-8.7,42.9) ; P=0.118$ \\
\hline Two previous CDI episodes & $8 / 10(80.0 ; 55.2,100.0)$ & $4 / 10(40.0 ; 9.6,70.4)$ & $40.0(0.8,79.2) ; P=0.141$ \\
\hline Cancer present & $23 / 38(60.5 ; 45.0,76.1)$ & $18 / 37(48.6 ; 32.5,64.8)$ & $11.9(-10.5,34.3) ; P=0.274$ \\
\hline Cancer absent & $101 / 139(72.7 ; 65.3,80.1)$ & $88 / 142(62.0 ; 54.0,70.0)$ & $10.7(-0.2,21.6) ; P=0.061$ \\
\hline PCR-RT 027 & $20 / 25(80.0 ; 64.3,95.7)$ & $9 / 22(40.9 ; 20.4,61.5)$ & $39.1(13.2,64.9) ; P=0.006$ \\
\hline Other RT & $104 / 152(68.4 ; 61.0,75.8)$ & $97 / 157(61.8 ; 54.2,69.4)$ & $6.6(-4.0,17.2) ; P=0.221$ \\
\hline
\end{tabular}

$C I$, confidence interval; EOT, end of treatment; $E P F X$, extended-pulsed fidaxomicin; $m F A S$, modified full analysis set (all patients with confirmed CDI who were randomised and received at least one dose of study medication); $P C R$, polymerase chain reaction; $R T$, ribotype. $P$ values were obtained from the Cochran-Mantel-Haenszel test, apart from the $P$ value for the difference in outcome for PCR-ribotype 027 versus other ribotypes, which was obtained from the chi-square test. Thirty days after EOT is day 55 for the EPFX arm and day 40 for the vancomycin arm 


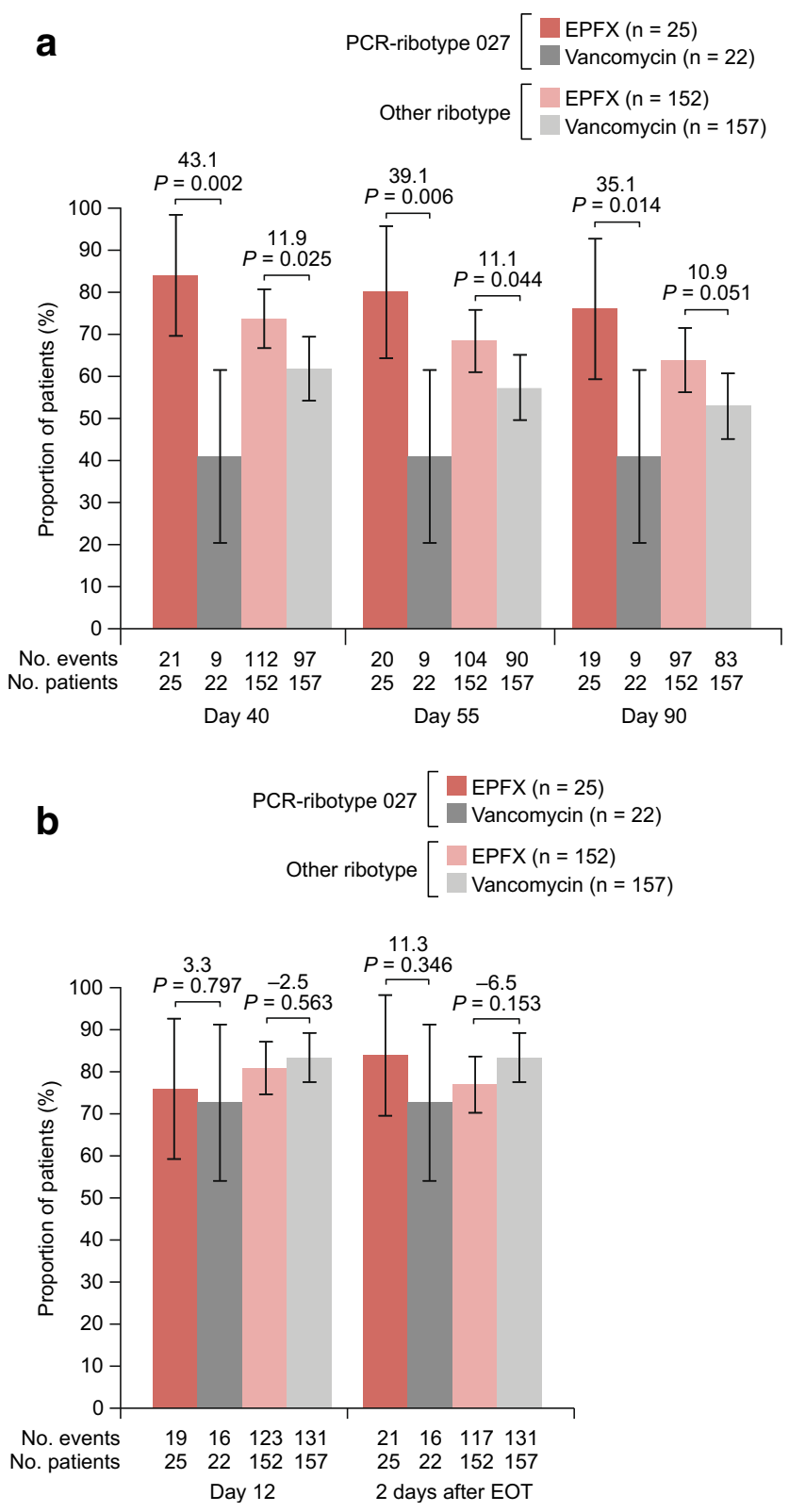

Fig. 2 Clinical outcomes of Clostridium difficile infection treatment with extended-pulsed fidaxomicin (EPFX) and vancomycin by presence of $C$. difficile PCR-ribotype 027 (present or absent), mFAS. a Sustained clinical cure (SCC) over time. b Clinical response. EOT, end of treatment; $m F A S$, modified full analysis set (all patients with confirmed CDI who were randomised and received at least one dose of study medication). $P$ values were obtained from the chi-square test. Two days after EOT is day 27 for the EPFX arm and day 12 for the vancomycin arm; 30 days after EOT is day 55 for the EPFX arm and day 40 for the vancomycin arm

one or two prior CDI episodes, and the correspondingly high within-sample variability.

Patients without cancer at baseline had significantly higher rates of SCC at days 40, 55 and 90 with EPFX versus vancomycin (Online Resource; ESM Fig. 4a). Other efficacy endpoints in patients with and without cancer did not differ between treatments (Online Resource; ESM Fig. 4a and 4b). These results contrast with those from a previous analysis by Cornely et al. in which the SCC rate was significantly higher with standard-regimen fidaxomicin than vancomycin $(73.6 \%$ versus $52.1 \% ; P=0.003)$ in 183 patients with $\mathrm{CDI}$ and cancer from two phase 3 studies [2]. This difference in outcome may be attributable to the smaller sample size of our study (75 patients with CDI and baseline cancer) and slightly greater median age (75 years for the overall population in our study versus 69 and 63 years in patients with and without cancer, respectively, in the Cornely et al. study [2]). Our findings aligned with those of the previous study in that patients with cancer had lower rates of initial clinical cure and SCC than patients without cancer, regardless of treatment choice.

C. difficile PCR-ribotype 027 was the most prevalent ribotype in a recent pan-European survey [23] and has been associated with outbreaks of increased severity [7] and greater risk of CDI recurrence than other strains [20]. In our study, rates of SCC at days 40, 55 and 90 were significantly higher with EPFX than with vancomycin in patients infected with $C$. difficile PCR-ribotype 027 (Fig. 2a). Rates of SCC at days 40 and 55 were significantly higher with EPFX than vancomycin in patients with other $C$. difficile PCR-ribotypes. No other significant between-treatment efficacy differences were observed in relation to $C$. difficile PCR-ribotype (Fig. 2a, b). In previous phase 3 registration studies, rates of recurrence were significantly lower in patients infected with non-PCRribotype 027 strains but not in patients infected with PCRribotype $027[14,15]$. Further studies would be required to provide analyses of sufficient power to permit robust conclusions on efficacy differences in relation to PCR-ribotype 027.

Of note, clinical response at day 12 and 2 days after EOT was numerically lower with EPFX than with vancomycin in the majority of subgroups investigated here, and in the overall mFAS population [17]. These results contrast with those of previous phase 3 trials $[14,15]$, in which standard-regimen fidaxomicin achieved numerically higher rates of clinical cure at 2 days after EOT, compared with standard vancomycin. The lower rate of initial clinical response with EPFX may be due to the administration of fidaxomicin on only alternate days after day 5, thus delaying the reduction in $C$. difficile count compared with vancomycin treatment.

\section{Recurrence of CDI}

Current ESCMID guidelines recommend that patients at risk of recurrent CDI are given standard-regimen fidaxomicin or vancomycin [5]. Standard-regimen fidaxomicin is associated with a lower recurrence rate than standard vancomycin [14, 15]; moreover, in the overall EXTEND population, EPFX showed even lower recurrence rates than previously observed 
Table 3 Recurrence of CDI at day 90 stratified by randomisation factors, mFAS

\begin{tabular}{|c|c|c|c|c|c|c|}
\hline \multirow[t]{2}{*}{ CDI severity } & \multirow[t]{2}{*}{ Cancer status } & \multirow[t]{2}{*}{ Age (years) } & \multirow{2}{*}{$\begin{array}{l}\text { Number of } \\
\text { previous occurrences }\end{array}$} & \multicolumn{2}{|c|}{ Recurrence of CDI $(\% ; 95 \% \mathrm{CI})$} & \multirow[t]{2}{*}{ Treatment difference $(95 \% \mathrm{CI})$} \\
\hline & & & & $\operatorname{EPFX}(N=138)$ & Vancomycin $(N=147)$ & \\
\hline \multirow[t]{12}{*}{ Severe } & \multirow[t]{6}{*}{ Presence } & \multirow[t]{3}{*}{$\geq 75$} & 2 & $0 / 1$ & $0 / 1$ & \\
\hline & & & 1 & & $0 / 2$ & \\
\hline & & & 0 & $0 / 5$ & $0 / 3$ & \\
\hline & & \multirow[t]{3}{*}{$60-74$} & 2 & & & \\
\hline & & & 1 & & $0 / 1$ & \\
\hline & & & 0 & $0 / 1$ & $1 / 5(20.0 ; 0.5,71.6)$ & $-20.0(-97.5,84.8)$ \\
\hline & \multirow[t]{6}{*}{ Absence } & \multirow[t]{3}{*}{$\geq 75$} & 2 & & $1 / 1(100.0 ; 2.5,100.0)$ & \\
\hline & & & 1 & $0 / 4$ & $1 / 2(50.0 ; 1.3,98.7)$ & $-50.0(-98.7,45.2)$ \\
\hline & & & 0 & $2 / 19(10.5 ; 1.3,33.1)$ & $5 / 24(20.8 ; 7.1,42.2)$ & $-10.3(-39.0,19.5)$ \\
\hline & & \multirow[t]{3}{*}{$60-74$} & 2 & & $1 / 1(100.0 ; 2.5,100.0)$ & \\
\hline & & & 1 & $1 / 1(100.0 ; 2.5,100.0)$ & & \\
\hline & & & 0 & $1 / 14(7.1 ; 0.2,33.9)$ & $4 / 10(40.0 ; 12.2,73.8)$ & $-32.9(-66.9,8.2)$ \\
\hline \multirow[t]{12}{*}{ Non-severe } & \multirow[t]{6}{*}{ Presence } & \multirow[t]{3}{*}{$\geq 75$} & 2 & $0 / 1$ & & \\
\hline & & & 1 & & & \\
\hline & & & 0 & $0 / 6$ & $5 / 8(62.5 ; 24.5,91.5)$ & $-62.5(-91.5,-10.1)$ \\
\hline & & \multirow[t]{3}{*}{$60-74$} & 2 & & & \\
\hline & & & 1 & $0 / 1$ & $0 / 2$ & \\
\hline & & & 0 & $0 / 9$ & $1 / 6(16.7 ; 0.4,64.1)$ & $-16.7(-64.1,36.2)$ \\
\hline & \multirow[t]{6}{*}{ Absence } & \multirow[t]{3}{*}{$\geq 75$} & 2 & $0 / 5$ & $1 / 3(33.3 ; 0.8,90.6)$ & $-33.3(-90.5,37.0)$ \\
\hline & & & 1 & $1 / 6(16.7 ; 0.4,64.1)$ & $2 / 6(33.3 ; 4.3,77.7)$ & $-16.7(-69.7,44.7)$ \\
\hline & & & 0 & $1 / 27(3.7 ; 0.1,19.0)$ & $3 / 30(10.0 ; 2.1,26.5)$ & $-6.3(-32.0,19.5)$ \\
\hline & & \multirow[t]{3}{*}{$60-74$} & 2 & $1 / 3(33.3 ; 0.8,90.6)$ & $0 / 1$ & $33.3(-80.9,97.5)$ \\
\hline & & & 1 & $1 / 8(12.5 ; 0.3,52.7)$ & $4 / 10(40.0 ; 12.2,73.8)$ & $-27.5(-66.8,19.3)$ \\
\hline & & & 0 & $2 / 27(7.4 ; 0.9,24.3)$ & $4 / 31(12.9 ; 3.6,29.8)$ & $-5.5(-30.5,20.2)$ \\
\hline
\end{tabular}

Includes only patients who had clinical response at 2 days after EOT

with the standard regimen [17]. However, there was no between-treatment difference in the incidence of recurrent CDI according to the subgroups investigated here, although numbers were too small to permit definitive conclusions (Table 3).

\section{Safety}

The safety profiles of EPFX and standard vancomycin were broadly similar within the subgroups analysed. A greater number of treatment-emergent adverse events, serious adverse events and deaths were reported in patients aged $\geq 75$ years in the vancomycin arm than in the EPFX arm (Table 4).

\section{Conclusions}

A strength of the present analysis was the inclusion of elderly patients (median age, 75 years), who are not typically enrolled in randomised, controlled trials because polypharmacy and multiple underlying conditions may obscure potential treatment benefits. However, we successfully demonstrate positive outcomes in this difficult-to-treat population. The extensive 90-day follow-up period of our study also provides considerable opportunity to assess long-term efficacy and safety.

Limitations of the study include the lack of tapered vancomycin or standard 10-day fidaxomicin regimens as comparators, which would have broadened the information available but restricted the feasibility of performing such a study. In addition, the post hoc analysis results in relation to PCRribotype were not corrected for multiple testing. However, the results obtained for this and other subgroups were consistent across the assessed endpoints, and adjustment for multiple testing was not expected to impact on proportions and confidence intervals, only $P$ values. When statistically significant, the latter were markedly lower than the $\leq 0.05$ threshold in most instances. Although the subgroups analysed here are small, the results suggest greater efficacy with EPFX versus vancomycin in some patient groups. Additionally, EPFX was 
Table 4 Treatment-emergent adverse events (based on MedDRA v14.1) during the study, by subgroup, SAF

TEAE frequency by subgroup $\operatorname{EPFX}(n=181)$ Vancomycin $(n=181)$

\begin{tabular}{|c|c|c|}
\hline \multirow{2}{*}{ Any TEAE } & \multicolumn{2}{|c|}{ Patients, $n(\%)$} \\
\hline & & \\
\hline All patients & $121(66.9)$ & $128(70.7)$ \\
\hline \multicolumn{3}{|l|}{ Age category } \\
\hline $60-74$ years & $56(30.9)$ & $53(29.3)$ \\
\hline$\geq 75$ years & $65(35.9)$ & 75 (41.4) \\
\hline \multicolumn{3}{|l|}{ CDI severity } \\
\hline Severe & $50(27.6)$ & $49(27.1)$ \\
\hline Non-severe & $71(39.2)$ & 79 (43.6) \\
\hline \multicolumn{3}{|c|}{ Prior CDI occurrence } \\
\hline None & 94 (51.9) & $101(55.8)$ \\
\hline One & $19(10.5)$ & $18(9.9)$ \\
\hline Two & $8(4.4)$ & $9(5.0)$ \\
\hline \multicolumn{3}{|c|}{ Cancer diagnosis } \\
\hline Present & $23(12.7)$ & $32(17.7)$ \\
\hline Absent & $98(54.1)$ & $96(53.0)$ \\
\hline \multicolumn{3}{|c|}{ Any serious TEAE ${ }^{\mathrm{a}}$} \\
\hline All patients & 68 (37.6) & $78(43.1)$ \\
\hline \multicolumn{3}{|l|}{ Age category } \\
\hline $60-74$ years & $33(18.2)$ & $30(16.6)$ \\
\hline$\geq 75$ years & $35(19.3)$ & $47(26.0)$ \\
\hline \multicolumn{3}{|l|}{ CDI severity } \\
\hline Severe & $33(18.2)$ & $33(18.2)$ \\
\hline Non-severe & $35(19.3)$ & $44(24.3)$ \\
\hline \multicolumn{3}{|c|}{ Prior CDI occurrence } \\
\hline None & $58(32.0)$ & $61(33.7)$ \\
\hline One & $7(3.9)$ & $11(6.1)$ \\
\hline Two & $3(1.7)$ & $5(2.8)$ \\
\hline \multicolumn{3}{|c|}{ Cancer diagnosis } \\
\hline Present & $17(9.4)$ & $21(11.6)$ \\
\hline Absent & $51(28.2)$ & $56(30.9)$ \\
\hline \multicolumn{3}{|c|}{ TEAE-related death ${ }^{\mathrm{b}}$} \\
\hline All patients & $28(15.5)$ & $36(19.9)$ \\
\hline Day 1-27 & $17(9.4)$ & $9(5.0)$ \\
\hline Day $27-95^{\mathrm{b}}$ & $12(7.3)$ & $27(15.7)$ \\
\hline \multicolumn{3}{|l|}{ Age category } \\
\hline $60-74$ years & $12(6.6)$ & $12(6.6)$ \\
\hline$\geq 75$ years & $16(8.8)$ & $24(13.3)$ \\
\hline \multicolumn{3}{|l|}{ CDI severity } \\
\hline Severe & $14(7.7)$ & $14(7.7)$ \\
\hline Non-severe & $14(7.7)$ & $22(12.2)$ \\
\hline \multicolumn{3}{|c|}{ Prior CDI occurrence } \\
\hline None & $25(13.8)$ & $32(17.7)$ \\
\hline One & $1(0.6)$ & $3(1.7)$ \\
\hline Two & $2(1.1)$ & $1(0.6)$ \\
\hline \multicolumn{3}{|c|}{ Cancer diagnosis } \\
\hline Present & $9(5.0)$ & $13(7.2)$ \\
\hline Absent & $19(10.5)$ & $23(12.7)$ \\
\hline
\end{tabular}

Results are given for the Safety Analysis Set: all patients who were randomised and received at least one dose of study medication. CDI, Clostridium difficile infection; TEAE, treatment-emergent adverse event. ${ }^{a}$ TEAE resulting in death, hospitalisation, persistent or significant disability, incapacity, congenital abnormality, or other medically important events; or considered to be life-threatening. ${ }^{\mathrm{b}}$ Includes one death in the EPFX arm not considered TEAE-related as it occurred after day 90

not associated with lower SCC rates or a higher incidence of adverse events, compared with standard vancomycin, in any patient subgroup investigated here.

In summary, subgroup analyses of the EXTEND study determined that an extended-pulsed fidaxomicin regimen is efficacious and well tolerated as a potential treatment for CDI regardless of age, presence of cancer, infection with $C$. difficile PCR-ribotype 027 , CDI severity or prior CDI episodes.

Acknowledgements The authors thank the EXTEND study group for their important contributions to this work. The study was initiated and funded by Astellas Pharma, Inc. Medical writing support was provided by Rhian Harper Owen, $\mathrm{PhD}$, for Cello Health MedErgy and funded by Astellas Pharma, Inc.

Author contributions Study design: OAC, MJGTV, NA, AG, AK Conduct and acquisition of data: OAC, MJGTV, NA, AG, AK, GK, BG Analysis and interpretation of data: OAC, MJGTV, NA, AG, AK, GK, BG Writing of the manuscript: OAC, MJGTV, NA, AG, AK, GK, BG

Funding This work was supported by Astellas Pharma, Inc.

Data availability Access to anonymised individual participant level data collected during the trial, in addition to supporting clinical documentation, is planned for trials conducted with approved product indications and formulations, as well as compounds terminated during the development. Conditions and exceptions are described under the Sponsor Specific Details for Astellas on www.clinicalstudydatarequest.com. Study-related supporting documentation is redacted and provided if available, such as the protocol and amendments, statistical analysis plan and clinical study report. Access to participant level data is offered to researchers after publication of the primary manuscript (if applicable) and is available as long as Astellas has legal authority to provide the data. Researchers must submit a proposal to conduct a scientifically relevant analysis of the study data. The research proposal is reviewed by an Independent Research Panel. If the proposal is approved, access to the study data is provided in a secure data sharing environment after receipt of a signed Data Sharing Agreement.

\section{Compliance with ethical standards}

Disclosure of potential conflict of interest $\mathrm{OAC}$ has received research grants from Actelion, Arsanis, Astellas, AstraZeneca, Basilea, Bayer, Cidara, Duke University, F2G, Gilead, GSK, Leeds University, Medicines Company, MedPace, Melinta Therapeutics, Merck/MSD, Miltenyi, Pfizer, Rempex, Roche, Sanofi Pasteur, Scynexis, Seres Therapeutics and The Medicines Company; and personal fees from Actelion, Amplyx, Astellas, Basilea, Cidara, Da Volterra, F2G, Gilead, IQVIA, Janssen Pharmaceuticals, Matinas, Menarini Ricerche, Merck/ MSD, Paratek Pharmaceuticals, Pfizer, PSI, Scynexis, Seres Therapeutics, Summit, Tetraphase and Vical.

MJGTV is a consultant to Astellas Pharma, Berlin Chemie, MaaT Pharma and MSD/Merck; has served at the speakers' bureaux of Astellas Pharma, Basilea, Falk Foundation, Gilead Sciences, Merck/ MSD, Organobalance and Pfizer and received research funding from 3M, Astellas Pharma, DaVolterra, Gilead Sciences, Merck/MSD, Morphochem, Organobalance and Seres Therapeutics.

NA was an employee of Astellas Pharma, Inc. during the conduct of the study.

AG is a full-time employee of Astellas Pharma Europe Ltd.

AK is a full-time employee of Astellas Pharma Ltd. and has patents WO2015169451 A1 and EP17167541.6 pending to Astellas Pharma Europe Ltd.

GK is a consultant statistician for Astellas Pharma, Inc.

BG received personal and institutional fees from Astellas Pharma, Pfizer and MSD, non-financial support from Astellas Pharma and research grants from Combioxin and Fondation Santos Suarez. 
Ethical approval The study was conducted in accordance with local ethical committees' regulations, the Declaration of Helsinki, and the International Council of Harmonisation Good Clinical Practice guidelines.

Informed consent Patients provided written informed consent and could withdraw from the study at any time.

Open Access This article is distributed under the terms of the Creative Commons Attribution 4.0 International License (http:// creativecommons.org/licenses/by/4.0/), which permits unrestricted use, distribution, and reproduction in any medium, provided you give appropriate credit to the original author(s) and the source, provide a link to the Creative Commons license, and indicate if changes were made.

\section{References}

1. Delgado A, Reveles IA, Cabello FT, Reveles KR (2017) Poorer outcomes among cancer patients diagnosed with Clostridium difficile infections in United States community hospitals. BMC Infect Dis 17:448. https://doi.org/10.1186/s12879-017-2553-Z

2. Cornely OA, Miller MA, Fantin B, Mullane K, Kean Y, Gorbach S (2013) Resolution of Clostridium difficile-associated diarrhea in patients with cancer treated with fidaxomicin or vancomycin. J Clin Oncol 31:2493-2499. https://doi.org/10.1200/JCO.2012.45.5899

3. Skoutelis A, Pefanis A, Tsiodras S, Sipsas NV, Lelekis M, Lazanas MC et al (2017) Point-prevalence survey of healthcare facility-onset healthcare-associated Clostridium difficile infection in Greek hospitals outside the intensive care unit: the C. DEFINE study. PLoS One 12: e0182799. https://doi.org/10.1371/journal.pone.0182799

4. Kamboj M, Son C, Cantu S, Chemaly RF, Dickman J, Dubberke E et al (2012) Hospital-onset Clostridium difficile infection rates in persons with cancer or hematopoietic stem cell transplant: a C3IC network report. Infect Control Hosp Epidemiol 33:1162-1165. https://doi.org/10.1086/668023

5. Debast S, Bauer M, Kuijper E (2014) European Society of Clinical Microbiology and Infectious Diseases: update of the treatment guidance document for Clostridium difficile infection. Clin Microbiol Infect 20:1-26. https://doi.org/10.1111/1469-0691.12418

6. See I, Mu Y, Cohen J, Beldavs ZG, Winston LG, Dumyati G et al (2014) NAP1 strain type predicts outcomes from Clostridium difficile infection. Clin Infect Dis 58:1394-1400. https://doi.org/ $10.1093 / \mathrm{cid} / \mathrm{ciu} 125$

7. Hensgens MPM, Goorhuis A, Dekkers OM, Van Benthem BHB, Kuijper EJ (2013) All-cause and disease-specific mortality in hospitalized patients with Clostridium difficile infection: a multicenter cohort study. Clin Infect Dis 56:1108-1116. https://doi.org/10. 1093/cid/cis1209

8. Kelly CP (2012) Can we identify patients at high risk of recurrent Clostridium difficile infection? Clin Microbiol Infect 18:21-27. https://doi.org/10.1111/1469-0691.12046

9. Petrella LA, Sambol SP, Cheknis A, Nagaro K, Kean Y, Sears PS et al (2012) Decreased cure and increased recurrence rates for Clostridium difficile infection caused by the epidemic $C$. difficile BI strain. Clin Infect Dis 55:351-357. https://doi.org/10.1093/cid/cis430

10. Lubbert C, Zimmermann L, Borchert J, Horner B, Mutters R, Rodloff AC (2016) Epidemiology and recurrence rates of Clostridium difficile infections in Germany: a secondary data analysis. Infect Dis Ther 5: 545-554. https://doi.org/10.1007/s40121-016-0135-9

11. Vardakas KZ, Polyzos KA, Patouni K, Rafailidis PI, Samonis G, Falagas ME (2012) Treatment failure and recurrence of Clostridium difficile infection following treatment with vancomycin or metronidazole: a systematic review of the evidence. Int $\mathrm{J}$ Antimicrob Agents 40:1-8. https://doi.org/10.1016/j.jantimicag.2012.01.004

12. Britton RA, Young VB (2012) Interaction between the intestinal microbiota and host in Clostridium difficile colonization resistance. Trends Microbiol 20:313-319. https://doi.org/10.1016/j.tim.2012. 04.001

13. Louie TJ, Cannon K, Byrne B, Emery J, Ward L, Eyben M et al (2012) Fidaxomicin preserves the intestinal microbiome during and after treatment of Clostridium difficile infection (CDI) and reduces both toxin re-expression and recurrence of CDI. Clin Infect Dis 55(Suppl 2):S132-S142. https://doi.org/10.1093/cid/cis338

14. Cornely OA, Crook DW, Esposito R, Poirier A, Somero MS, Weiss $\mathrm{K}$ et al (2012) Fidaxomicin versus vancomycin for infection with Clostridium difficile in Europe, Canada, and the USA: a doubleblind, non-inferiority, randomised controlled trial. Lancet Infect Dis 12:281-289. https://doi.org/10.1016/S1473-3099(11)70374-7

15. Louie TJ, Miller MA, Mullane K, Weiss K, Lentnek A, Golan Y et al (2011) Fidaxomicin versus vancomycin for Clostridium difficile infection. N Engl J Med 364:422-431. https://doi.org/10. 1056/NEJMoa0910812

16. Chilton $\mathrm{CH}$, Crowther GS, Todhunter SL, Ashwin H, Longshaw CM, Karas A et al (2015) Efficacy of alternative fidaxomicin dosing regimens for treatment of simulated Clostridium difficile infection in an in vitro human gut model. J Antimicrob Chemother 70:25982607. https://doi.org/10.1093/jac/dkv156

17. Guery B, Menichetti F, Anttila V, Adomakoh N, Aguado JM, Bisnauthsing $\mathrm{K}$ et al (2017) Extended-pulsed fidaxomicin versus vancomycin for Clostridium difficile infection in patients 60 years and older (EXTEND): a randomised, controlled, open-label, phase 3b/4 trial. Lancet Infect Dis 18:296-307. https://doi.org/10.1016/ S1473-3099(17)30751-X

18. Stubbs SLJ, Brazier JS, O’Neill GL, Duerden BI (1999) PCR targeted to the 16S-23S rRNA gene intergenic spacer region of Clostridium difficile and construction of a library consisting of 116 different PCR ribotypes. J Clin Microbiol 37:461-463

19. Janezic S, Indra A, Allerberger F, Rupnik M (2011) Use of different molecular typing methods for the study of heterogeneity within Clostridium difficile toxinotypes V and III. J Med Microbiol 60: 1101-1107. https://doi.org/10.1099/jmm.0.031054-0

20. Louie TJ, Miller MA, Crook DW, Lentnek A, Bernard L, High KP et al (2013) Effect of age on treatment outcomes in Clostridium difficile infection. J Am Geriatr Soc 61:222-230. https://doi.org/ 10.1111/jgs. 12090

21. Khanafer N, Vanhems P, Barbut F, Luxemburger C (2017) Factors associated with Clostridium difficile infection: a nested case-control study in a three year prospective cohort. Anaerobe 44:117-123. https://doi.org/10.1016/j.anaerobe.2017.03.003

22. Cornely OA, Miller MA, Louie TJ, Crook DW, Gorbach SL (2012) Treatment of first recurrence of Clostridium difficile infection: fidaxomicin versus vancomycin. Clin Infect Dis 55:S154-S161. https://doi.org/10.1093/cid/cis462

23. Davies KA, Ashwin H, Longshaw CM, Burns DA, Davis GL, Wilcox MH (2016) Diversity of Clostridium difficile PCR ribotypes in Europe: results from the European, multicentre, prospective, biannual, point-prevalence study of Clostridium difficile infection in hospitalised patients with diarrhoea (EUCLID), 2012 and 2. Euro Surveill 21:30294. https://doi.org/10.2807/1560-7917.ES.2016.21. 29.30294

Publisher's note Springer Nature remains neutral with regard to jurisdictional claims in published maps and institutional affiliations. 